\title{
Longitudinal analysis of cell-free mutated KRAS and CA 19-9 predicts survival following curative resection of pancreatic cancer
}

Saskia Hussung ${ }^{1,2}$, Dilara Akhoundova ${ }^{2}$, Julian Hipp ${ }^{3}$, Marie Follo ${ }^{1}$, Rhena F. U. Klarr ${ }^{1}$ Ulrike Philipp ${ }^{1}$, Florian Scherer ${ }^{1}$, Nikolas von Bubnoff ${ }^{1}$, Justus Duyster ${ }^{1,4,5}$, Melanie Boerries ${ }^{4,5,6,7}$, Uwe Wittel ${ }^{3}$ and Ralph M. Fritsch ${ }^{1,2,5^{*}}$ (i)

\begin{abstract}
Background: Novel biomarkers and molecular monitoring tools hold potential to improve outcome for patients following resection of pancreatic ductal adenocarcinoma (PDAC). We hypothesized that the combined longitudinal analysis of mutated cell-free plasma KRAS (cfKRAS ${ }^{m u t}$ ) and CA 19-9 during adjuvant treatment and follow-up might more accurately predict disease course than hitherto available parameters.
\end{abstract}

Methods: Between 07/2015 and 10/2018, we collected 134 plasma samples from 25 patients after R0/R1-resection of PDAC during adjuvant chemotherapy and post-treatment surveillance at our institution. Highly sensitive discriminatory multi-target ddPCR assays were employed to screen plasma samples for cfKRAS ${ }^{\text {mut }}$. cfKRAS ${ }^{\text {mut }}$ and CA 19-9 dynamics were correlated with recurrence-free survival (RFS) and overall survival (OS). Patients were followedup until 01/2020.

Results: Out of 25 enrolled patients, $76 \%$ had undergone R0 resection and $48 \%$ of resected PDACs were pN0. 17/ 25 (68\%) of patients underwent adjuvant chemotherapy. Median follow-up was 22.0 months, with 19 out of 25 (76\%) patients relapsing during study period. Median RFS was 10.0 months, median OS was 22.0 months. Out of clinicopathologic variables, only postoperative CA 19-9 levels and administration of adjuvant chemotherapy correlated with survival endpoints. cfKRAS ${ }^{\text {mut. }}$ was detected in 12/25 (48\%) of patients, and detection of high levels inversely correlated with survival endpoint. Integration of cfKRAS ${ }^{m u t}$ and CA 19-9 levels outperformed either individual marker. cfKRAS ${ }^{\text {mut }}$ outperformed CA 19-9 as dynamic marker since increase during adjuvant chemotherapy and follow-up was highly predictive of early relapse and poor OS.

Conclusions: Integrated analysis of cfKRAS ${ }^{m u t}$ and CA 19-9 levels is a promising approach for molecular monitoring of patients following resection of PDAC. Larger prospective studies are needed to further develop this approach and dissect each marker's specific potential.

Keywords: Liquid biopsy, Droplet digital PCR (ddPCR), Cell-free DNA (cfDNA), Circulating KRAS (cfKRAS mut), Prognostic biomarkers, Pancreatic cancer, Molecular monitoring

\footnotetext{
* Correspondence: ralph.fritsch@usz.ch

'Department of Medicine I (Hematology, Oncology and Stem Cell

Transplantation), Freiburg University Medical Center, Freiburg, Germany

${ }^{2}$ Department of Medical Oncology and Hematology, Zurich University

Hospital, Raemistrasse 100, 8091 Zürich, Switzerland

Full list of author information is available at the end of the article
}

(c) The Author(s). 2021 Open Access This article is licensed under a Creative Commons Attribution 4.0 International License, which permits use, sharing, adaptation, distribution and reproduction in any medium or format, as long as you give appropriate credit to the original author(s) and the source, provide a link to the Creative Commons licence, and indicate if changes were made. The images or other third party material in this article are included in the article's Creative Commons licence, unless indicated otherwise in a credit line to the material. If material is not included in the article's Creative Commons licence and your intended use is not permitted by statutory regulation or exceeds the permitted use, you will need to obtain permission directly from the copyright holder. To view a copy of this licence, visit http://creativecommons.org/licenses/by/4.0/ The Creative Commons Public Domain Dedication waiver (http://creativecommons.org/publicdomain/zero/1.0/) applies to the data made available in this article, unless otherwise stated in a credit line to the data. 


\section{Background}

Despite significant progress in understanding tumor genetics and the molecular mechanisms driving tumor development and resistance to therapy, only minor improvements have been achieved to date in the treatment of patients with pancreatic ductal adenocarcinoma (PDAC). With an average 5-year overall survival (OS) rate of only $10 \%$ across all stages, most patients still succumb to their disease, making PDAC one of the most aggressive tumor entities [1-3]. The only potentially curative treatment is surgical resection of early-stage tumors $[4,5]$. However, recurrence rates even after R0 resection remain unacceptably high [6-10]. The integration of more efficacious systemic chemotherapy regimens has improved median overall survival [11], yet responses of individual PDACs to chemotherapy are highly heterogeneous and personalization of perioperative therapy is in its infancy [12-18] .

Consequently, the development and validation of novel biomarkers and molecular monitoring tools to predict disease course and assess efficacy of adjuvant chemotherapy are urgently needed. The analysis of tumor-derived cellfree nucleic acids (ctDNA) extracted from the plasma and other body fluids is a promising tool for molecular diagnostics and non-invasive monitoring of cancer patients [19-26]. Up to 95\% of PDACs harbor activating hot spot mutations in KRAS which are readily detectable in the circulation of PDAC patients [20, 27-29]. We recently described the development and validation of highly sensitive single-target and discriminatory multi-target KRAS ddPCR assays for the analysis of cfDNA [30]. These assays allow identification and quantification of mutated KRAS directly from circulation without previous knowledge of tumor KRAS mutational status, which is not routinely tested for resectable PDACs.

For this study, we hypothesized that longitudinal

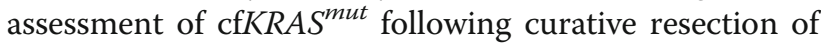
PDAC in combination with established protein biomarkers might better identify patients at risk for imminent tumor relapse, indicate failure of adjuvant treatment and ultimately guide treatment according to molecular monitoring. To study the feasibility of this approach, we analyzed plasma samples collected from patients undergoing adjuvant chemotherapy and posttreatment surveillance at our institution in a singlecenter retrospective biomarker study aiming to identify associations between cfKRAS ${ }^{\text {mut }}$ and CA 19-9 dynamics and clinical outcome post PDAC resection.

\section{Methods}

\section{Study design and population}

25 patients with resected pancreatic adenocarcinoma (following R0 or R1 curative-intended resection) were included in a retrospective observational single center biomarker study conducted at Freiburg University Medical Center. Local institutional review board (IRB) approved all study procedures (EK48/18). All patients provided written informed consent for sample collection and analysis. 17/25 patients underwent adjuvant chemotherapy (4/17 Gemcitabine, 4/17 FOLFIRINOX, 8/17 Gemcitabine/Capecitabine, 1/17 Gemcitabine/nab-Paclitaxel). Further inclusion criteria were "collection of first sample within 8 weeks after resection", and "availability of plasma samples for cfDNA extraction". Key exclusion criteria included " $\mathrm{R} 2$ resection, evidence of metastatic disease on pre- or postoperative $\mathrm{CT}$ staging, histologies other than adenocarcinoma”. According to UICC/AJCC, $\mathrm{R} 0$ resection was defined as microscopic edge-negative resection, in which no microscopic residual tumor remains. R1 resection was defined as a microscopic residual tumor and R2 as macroscopically visible residual tumor. Primary endpoint was detection of $\mathrm{cf} K R A S^{\text {mut }}$ in at least one sample during study period. Secondary endpoints included association between changes in cfKRASmut and relapse-free survival (RFS) and overall survival (OS). Additionally, clinical, pathologic, treatment- and outcome-related data were analyzed.

\section{Collection of patient samples and CA 19-9 analysis}

Blood samples were collected at a median of 40 days (95\% CI 26-50) after resection, prior to adjuvant chemotherapy and during 3-monthly routine clinical follow-up visits. CA 19-9 measurements were performed at our center's fully certified clinical chemistry facilities. The threshold value for CA $19-9$ positivity was $36 \mathrm{U} / \mathrm{ml}$.

\section{Extraction of cell-free DNA (cfDNA) from plasma samples}

Blood samples were collected using commercially available EDTA tubes and plasma was extracted and frozen within one hour of collection. Plasma was extracted through two subsequent centrifugation steps at 3000 $\mathrm{rpm}$ and $14,000 \mathrm{rpm}$, each for $10 \mathrm{~min}$ at $4{ }^{\circ} \mathrm{C}$. Obtained plasma was stored at $-80^{\circ} \mathrm{C}$ until extraction of cfDNA. cfDNA was extracted from $4 \mathrm{ml}$ plasma following the SEP/SBS protocol of the PME-free circulating DNA extraction kit (Analytik Jena, cat. no. 845-IR-0003050), following manufacturer's instructions. Two subsequent elution steps with each $30 \mu \mathrm{l}$ Elution Buffer were performed to optimize the yield of extracted cfDNA. DNA was stored at $-20^{\circ} \mathrm{C}$ until cfDNA quantification. cfDNA was evaluated with fragment analyzer and quantified using Qubit 2.0 fluorometer. In patients with resectable PDAC, DNA yield from $4 \mathrm{ml}$ of plasma typically ranged from 1 to $20 \mathrm{ng} / \mu \mathrm{l}$.

\section{Droplet digital PCR (ddPCR)}

ddPCR for $\operatorname{cfKRAS} S^{\text {mut }}$ was performed as recently described [29]. Locked nucleic acid (LNA) probes and 
corresponding primer pairs for KRAS mutations were designed using Beacon Designer v.8.20 software (Premier Biosoft, Palo Alto, California, USA) and manufactured by Integrated DNA Technologies (IDT, Inc., Coralville, Iowa, USA). Wild type (WT) probes were labelled with hexachlorofluorescein (HEX), mutant (MUT) probes with 6-carboxyfluorescein (FAM). Primer and Probe sequences are listed in Supplemental Table 1. Primers, probes, template DNA and nuclease-free water (Ambion, Austin, TX) were added to ddPCR Supermix for Probes (Bio-Rad, cat. no \#186-3024). Reaction mix was set up as recommended. $20 \mu \mathrm{l}$ of this reaction mix along with $70 \mu \mathrm{l}$ reader oil were transferred into cartridges of a QX100/200TM Droplet Generator (Bio-Rad, cat. no. \#1863002) following manufacturer's instructions. All samples were assayed in quadruplicates. Droplets were generated, transferred into a 96-well PCR plate (Bio-Rad, cat. no. \#12001925) and PCR was then run on a C1000 Touch $^{\text {tw }}$ Thermal Cycler (Bio-Rad, cat. no. \#1851197). Finally, samples were analyzed on a QX100/200TM Droplet Reader (Bio-Rad, cat. no. 1863003) using QuantaSoft v1.7.4.0917 (Bio-Rad, cat.no. \#1864011). Internal ddPCR controls were carried out as previously published [29].

The absolute number of copies per milliliter of blood were calculated as follows: Copies $/ \mathrm{mL}$ plasma $=($ copies per $\mu \mathrm{L}$ of reaction as per QuantaSoft analysis software version 1.7.4.0917) $\times($ volume of ddPCR reaction $) \times([$ volume eluted/volume of DNA used in reaction]/volume of plasma used for cfDNA extraction). Mutant allele frequency was calculated as: Mutant allele frequency $=m u-$ tant copies $/ \mathrm{mL}$ of plasma / (mutant copies $/ \mathrm{mL}$ of plasma + wild-type copies/mL of plasma).

Limit of detection (LOD) and limit of blank (LOB) of the individual assays have been previously described [30]

In brief, cfDNA was screened for the presence of the 11 most commonly found KRAS hot spot mutations, in PDAC, covering more than $90 \%$ of PDAC cases. Highly sensitive single-target assays were used to confirm presence of the mutation identified.

\section{Statistical analysis}

Recurrence-free survival (RFS) was defined as time from resection of PDAC to the first radiologic recurrence (local or distant) or death due to PDAC. Overall survival (OS) was defined as time from the date of diagnosis until death due to any cause. The Kaplan-Meier survival analysis was performed to calculate both RFS and OS. Univariate analyses were performed using the log-rank test. In order to explore independent prognostic factors for RFS and OS, we used backward stepwise Cox regression modeling to estimate hazard ratio (HR) with 95\% confidence interval (CI). To compare independent variables, Chi-squared or Fisher's exact test and the
Mann-Whitney (rank-sum) test were performed. All statistical analyses were performed using GraphPad Prism Version 5.03 (GraphPad Software, Inc., La Jolla, California, USA) and SPSS 25 software Version 1.0.0.1327 (IBM Corporation, New York, United States). $P$ values $<0.05$ were considered as statistically significant.

\section{Results}

\section{Patient cohort}

25 patients with non-metastatic, R0/R1-resected adenocarcinoma of the pancreas were included in the study. Patient characteristics are summarized in Table 1. R0 resection rate was $76 \%(19 / 25), 12 / 25(48 \%)$ of tumors were nodal negative (pN0). 17/25 (68\%) patients underwent adjuvant chemotherapy. Median follow-up for the cohort was 22.0 months, with 19 out of 25 (76\%) patients relapsing during this period. Median RFS for the cohort was 10.0 months, median OS was 22.0 months. We performed univariate and multivariate survival analyses (Supplemental Tables 2 and 3, Figure S1) for established clinicopathologic variables. We found no significant correlation between R0 vs R1 resection and RFS or OS (Figure S1 A, B). However, we identified a significant inverse correlation between elevated CA 19-9 in the first sample collected after resection and RFS and OS (Figure S1 C, D) and significantly better OS (Figure S1 F) but not RFS (Figure S1 E) for patients undergoing adjuvant chemotherapy.

\section{Analysis of plasma cfKRAS ${ }^{\text {mut }}$}

We analyzed 134 plasma samples collected from 25 patients at routine follow-ups before, during and after adjuvant chemotherapy. First samples were taken at a median of 40 days (95\% CI 26-50) after resection prior to adjuvant chemotherapy. Median number of samples collected was 4 samples per patient (95\% CI 3-5 samples). Median time interval between sampling was 70 days (95\% CI 63-91). We screened cfDNA extracted from plasma samples for the presence of cfKRAS $^{\text {mut }}$ with recently described discriminatory multi-target KRAS ddPCR assays, covering the 11 most common KRAS hot spot mutations in PDAC [30]. At the postoperative stage, no molecular pathology data was available for any tumor. However, for a subset of patients, KRAS mutational status became available at relapse (Supplemental Table 4).

Across all samples analyzed, cfKRAS ${ }^{\text {mut }}$ was detected in $34 / 134$ (25\%) samples and $12 / 25(48 \%)$ of patients for at least one time point. In 14/15 (93.33\%) patients with later on determined tumor tissue KRAS mutational status, the SNV detected by ddPCR in plasma (cfKRAS ${ }^{\text {mut }}$ ) at any time point matched the KRAS SNV detected in tissue analysis (Supplemental Table 4), confirming the 
Table 1 Patient and tumor characteristics

\begin{tabular}{|c|c|}
\hline Clinicopathologic features & $n=25(\%)$ \\
\hline Median age (years) & 75 \\
\hline Age range & $42-81$ \\
\hline \multicolumn{2}{|l|}{ Sex } \\
\hline Male & $18(72)$ \\
\hline Female & $7(28)$ \\
\hline \multicolumn{2}{|l|}{ Tumor location } \\
\hline Pancreas head & $20(80)$ \\
\hline Pancreas body \& tail & $5(20)$ \\
\hline \multicolumn{2}{|l|}{ T stage } \\
\hline $\mathrm{T} 1-\mathrm{T} 2$ & $7(28)$ \\
\hline T3 & $18(72)$ \\
\hline \multicolumn{2}{|l|}{$\mathrm{N}$ status } \\
\hline NO & $12(48)$ \\
\hline$N 1-2$ & $13(52)$ \\
\hline \multicolumn{2}{|l|}{ R status } \\
\hline Ro & $19(76)$ \\
\hline R1 & $5(20)$ \\
\hline Rx & $1(4)$ \\
\hline \multicolumn{2}{|l|}{ Lymphovascular invasion } \\
\hline LO & $15(60)$ \\
\hline L1 & $10(40)$ \\
\hline \multicolumn{2}{|l|}{ Perineural invasion } \\
\hline Pno & $2(8)$ \\
\hline Pn1 & $23(92)$ \\
\hline \multicolumn{2}{|l|}{ Grading } \\
\hline G2 & $13(52)$ \\
\hline G3 & $12(48)$ \\
\hline \multicolumn{2}{|l|}{ Adjuvant chemotherapy } \\
\hline Yes & $17(68)$ \\
\hline No & $8(32)$ \\
\hline \multicolumn{2}{|l|}{ Time to relapse (months) } \\
\hline Median & 10 \\
\hline Range & $0.5-42$ \\
\hline \multicolumn{2}{|l|}{ Overall survival (months) } \\
\hline Median & 22 \\
\hline Range & $0.5-46$ \\
\hline
\end{tabular}

validity of ddPCR cfKRAS ${ }^{\text {mut }}$ analysis. In 0/134 (0\%) of plasma samples, a concurrent second KRAS SNV could be detected above assay threshold.

\section{Association of cfKRAS ${ }^{\text {mut }}$ and elevated CA 19-9 levels with survival endpoints}

Detection of cfKRAS ${ }^{\text {mut }}$ at any time point during study course above assay threshold was not associated with RFS or OS (Fig. 1a, b). However, post-hoc analysis uncovered that a more stringent cut-off level of 15 copies $K R A S^{\text {mut }}$ per ml plasma for $\operatorname{cf} K R A S^{\text {mut }}$ detected at any time point during study period was strongly associated with early relapse and poor survival (Fig. 1c, d). Analogously, when analyzing KRAS variant allele frequencies (VAF) instead of DNA copy numbers, detection of $\operatorname{cfKRAS}{ }^{\text {mut }}$ above a threshold of $0.5 \%$ VAF, as determined by post hoc analysis, was associated with inferior RFS and OS (Supplemental Figure 2 A, B). Notably, all $5 / 25$ patients with a copy number of 15 copies $K R A S^{\text {mut }}$ per $\mathrm{ml}$ or higher also had a VAF above $0.5 \%$, while $18 / 20$ patients with a copy number $<15$ copies $K R A S^{\text {mut }}$ per $\mathrm{ml}$ had a VAF $<0.5 \%$, suggesting large overlap between the two distinct ways of analysis. CA 19-9 levels were determined from the same blood collections. 12/25 (48\%) of patients had at least one blood sample with CA 19-9 above normal range during study course. Increased CA 19-9 at any time point was associated with significantly inferior RFS and a non-significant trend towards inferior OS (Fig. 1e, f). Notably, only 6/12 (50\%) patients were double positive for $\mathrm{cf} K R A S^{\text {mut }}$ and CA 19-9, indicating that $\mathrm{cfKRAS^{ \text {mut } }}$ and CA 19-9 positivity are not redundant. Patients with either CA 19-9 positivity or $\operatorname{cfKRAS^{\text {mut}}}$ levels $>15$ copies $/ \mathrm{mL}$ during study course $(14 / 25,56 \%)$ showed inferior RFS and OS, indicating that the integration of both biomarkers might be predictive and prognostic for a larger group of patients than assaying them individually (Fig. $1 \mathrm{~g}$, h). Survival of double positive patients was similar to single positive patients in our cohort (data not shown). When analyzing associations between liquid biomarkers and clinicopathologic variables, there was no significant correlation between $\mathrm{R}$ status and postoperative levels of $\mathrm{CA}$ 19-9, cfKRAS ${ }^{\text {mut }}$ or total cell-free DNA (cfDNA) concentrations (Supplemental Figure 2 C, D, E).

\section{Association of cfKRAS ${ }^{\text {mut }}$ and CA 19-9 dynamics with survival}

Protein tumor markers and cfDNA are highly dynamic biomarkers for the molecular monitoring of disease course and treatment response. We therefore next analyzed whether changes over time in either biomarker are associated with outcome in our cohort. For each 9/18 (50\%) patients with a sufficient number of follow-up samples, cfKRAS ${ }^{\text {mut }}$ or CA 19-9 levels increased during observation period. Increase of cfKRAS ${ }^{\text {mut }}$ or CA 19-9 during observation period was defined as numerical increase of the respective parameter in initially positive patients or rise above threshold in initially negative patients. Increase of $\mathrm{cf} K R A S^{\mathrm{mut}}$ was associated with significantly reduced OS (Fig. 2a), while increase of CA 19-9 was not significantly associated inferior OS (Fig. 2b). Similarly, early increase of $\mathrm{cf} K R A S^{\mathrm{mut}}$, defined as increase within 6 months after surgery, was strongly 
A

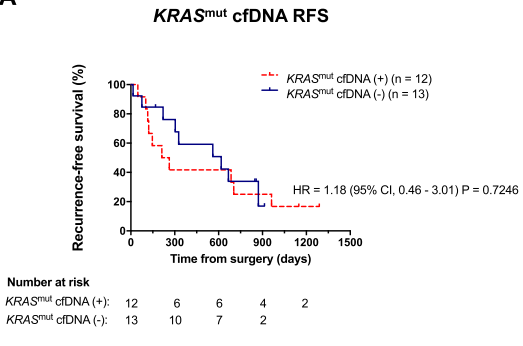

C
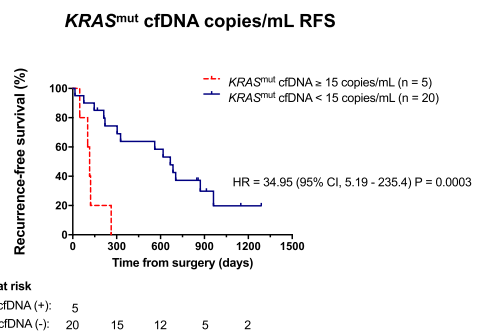

E

CA 19-9 RFS

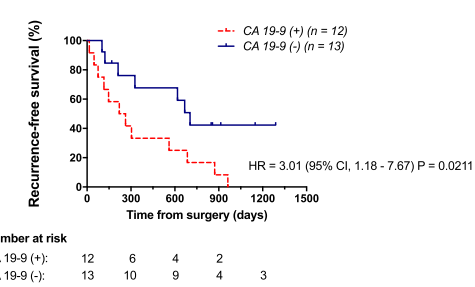

G

Combined KRAS ${ }^{\text {mut }}$ cfDNA + CA 19-9 RFS

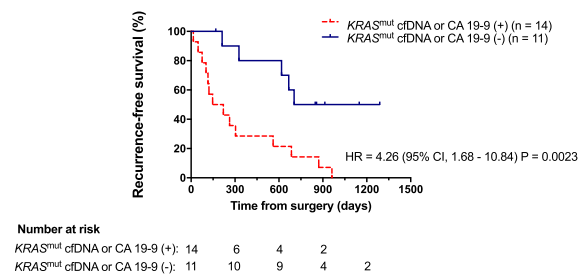

B

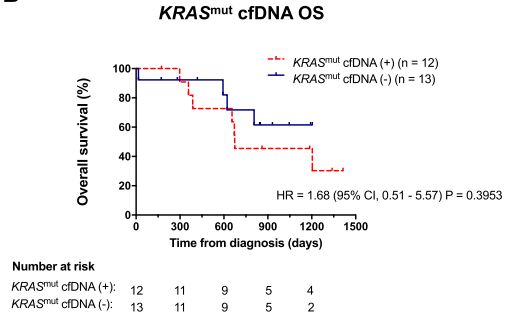

D
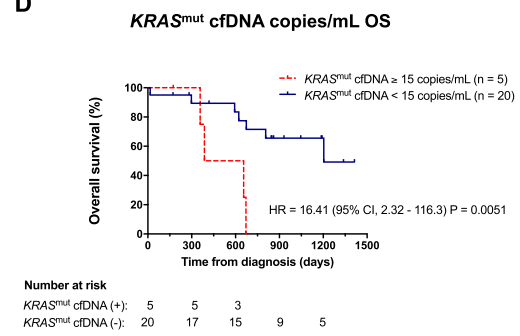

F

CA 19-9 OS

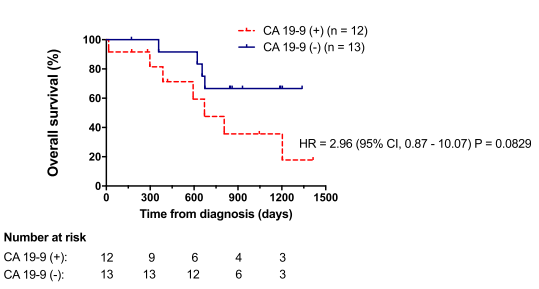

H

Combined KRAS ${ }^{\text {mut }}$ cfDNA + CA 19-9 OS

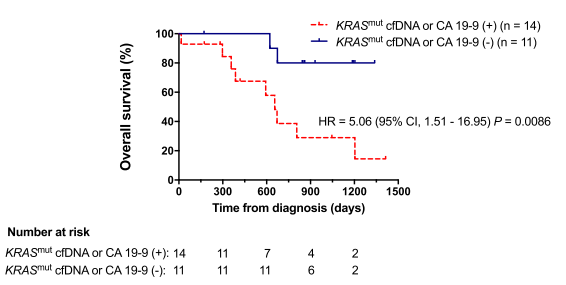

Fig. 1 Association of CFRAS ${ }^{m u t}$ detection and elevated CA 19-9 levels with survival endpoints. a, b Kaplan-Meier estimates of RFS (a) and OS (b) for patients following curative resection of PDAC with versus without detectable cfKRAS ${ }^{\text {mut }}$ at any time point during study period. $\mathbf{c}, \mathbf{d}$. A more stringent cfKRAS ${ }^{\text {mut }}$ cut-off level of $>15$ copies $/ \mathrm{mL}$ plasma was chosen. e, $\mathbf{f}$ Kaplan-Meier estimates of RFS (e) and OS (f) for resected PDAC patients with elevated $(>36 \mathrm{U} / \mathrm{mL}$ ) versus normal $(\leq 36 \mathrm{U} / \mathrm{mL})$ CA 19-9 levels at any time point during observation period. g, $\mathbf{h}$ Kaplan-Meier estimates of RFS $(\mathbf{g})$ and OS (h) for resected PDAC patients with either CA 19-9 positivity or cfKRAS mut levels $>15$ copies/mL cfKRAS during study course. OS, overall survival; RFS, recurrence-free survival; PDAC, pancreatic ductal adenocarcinoma

associated with inferior OS while early CA 19-9 increase was not significantly associated with shorter OS (Fig. 2c, d). Integrating both markers for the analysis of dynamic changes over time did not outperform cfKRAS ${ }^{\text {mut }}$ alone (Fig. 2e, f), suggesting that $\mathrm{cfKRAS^{ \textrm {mut } }}$ might be the biomarker of choice for longitudinal monitoring in this setting.

\section{Single patient analysis}

Figure 3 illustrates the relationship between $\mathrm{cfKRAS}{ }^{\text {mut }}$ and CA 19-9 (Fig. 3a) dynamics and tumor relapse for individual patients. $13 / 18$ patients in the analysis relapsed during observation period. Increase of $\mathrm{cfKR} A S^{\mathrm{mut}}$ or CA 19-9 was significantly associated with relapse. 09/ 13 patients with relapse during observation period showed an increase in either CA 19-9 or cfKRAS ${ }^{\text {mut }}$ (Fig. 3a), 6 out of these 9 patients showed an increase for both markers indicating partial overlap (not shown). Notably, 2/5 patients with no relapse during observation period still showed an increase in either CA 19-9 or cfKRAS ${ }^{\text {mut }}$ suggesting either insufficient duration of follow-up or suboptimal specificity when integrating 


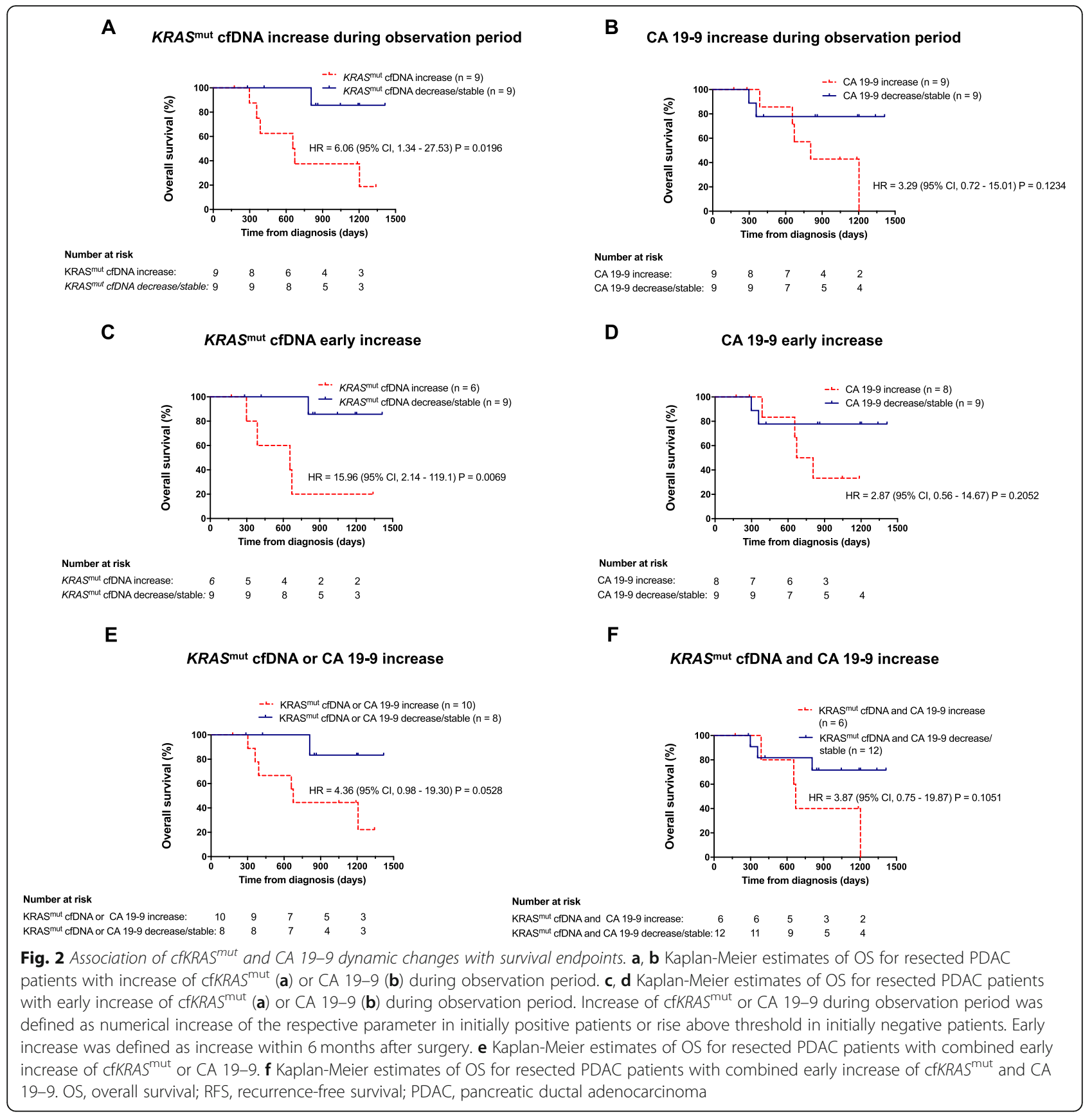

both markers for analysis. Single-patient analysis also illustrates dynamic changes during adjuvant chemotherapy and follow-up with several patients showing transient increases followed by decreases of either marker. Figure $3 \mathrm{~b}$ illustrates that, in most patients, relapsed was proceeded by a strong increase of CA 19-9 or cfKRASmut. However, single patient analyses also suggests that both cfKRAS ${ }^{\text {mut }}$ and CA 19-9 are highly dynamic biomarkers and that individual patterns can be highly heterogeneous requiring well-defined cut-off levels and extensive clinical validation for future clinical application.

\section{Discussion}

In an exploratory analysis, we followed a small cohort of pancreatic cancer patients after curative resection of pancreatic adenocarcinoma through adjuvant therapy and post-treatment follow-up. We analyzed mutated KRAS in cell-free DNA with discriminatory ddPCR assays and integrated results with CA 19-9 levels for 
A KRAS ${ }^{\text {mut }}$ cfDNA kinetics during observation period

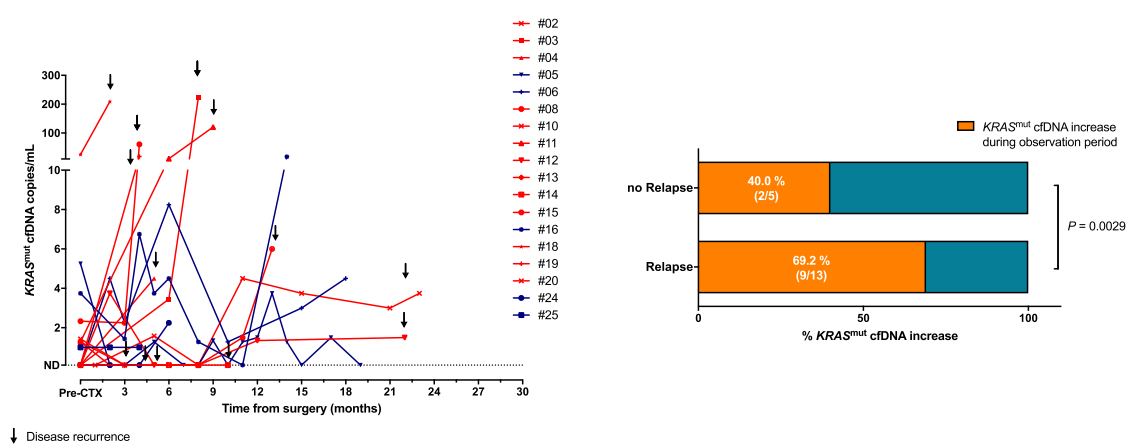

CA 19-9 kinetics during observation period

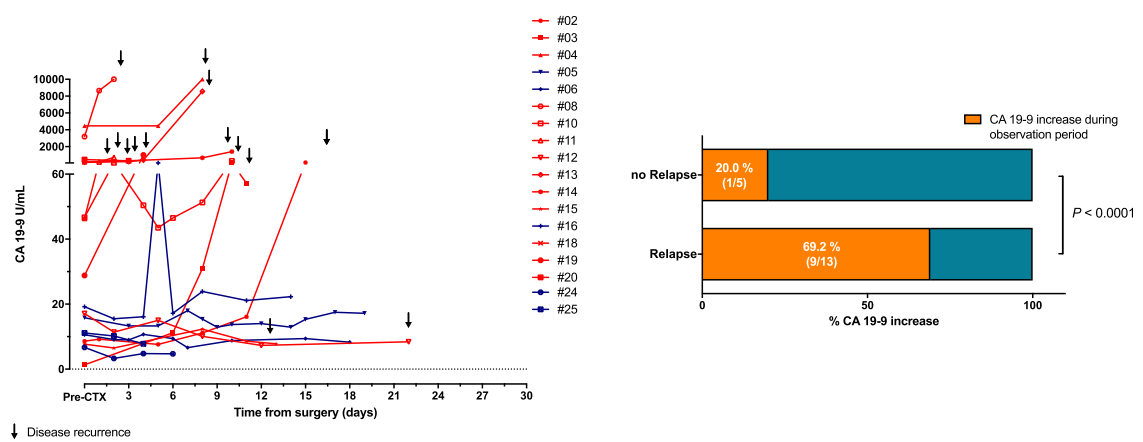

B

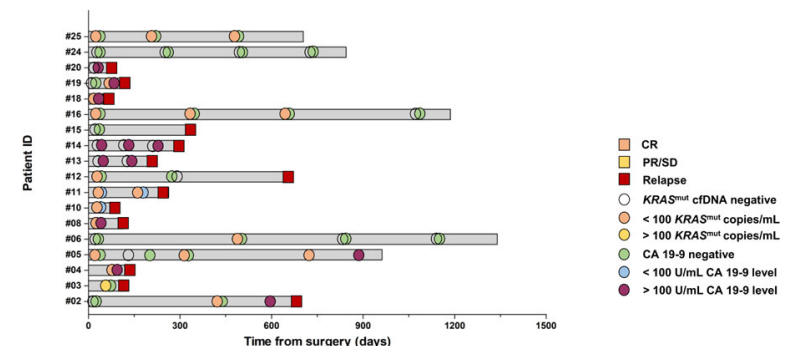

Fig. 3 Longitudinal cfKRAS ${ }^{\text {mut }}$ and CA 19-9 monitoring. a Top left: Absolut levels of cfKRAS ${ }^{\text {mut }}$ during observation period. Patients with relapse during study period are marked red. Black arrow mark the time of disease recurrence. Top right: Relapse versus non-relapse patients with increase in cfKRAS ${ }^{\text {mut }}$ during observation period. Fisher's exact test was used to test for statistical significance between the two groups. $P$ values $<0.05$ were considered significant. Bottom left: Absolut levels of CA 19-9 during observation period. Patients with relapse are marked red. Black arrows mark the time of disease recurrence. Bottom right: Relapse versus non-relapse patients with increase in cfKRAS ${ }^{\text {mut }}$ during observation period. Fisher's exact test was used to test for statistical significance between the two groups. $P$ values $<0.05$ were considered significant. $\mathbf{b}$ Swimmers plot of disease course of resected PDAC patients. cfKRAS ${ }^{\text {mut }}$ and CA 19-9 analysis in blood were compared to clinical course of disease before and during adjuvant chemotherapy. CR, complete response; PD, progressive disease; PR, partial response; SD, stable disease

association with relapse and survival endpoints. Numerous studies have unveiled the potential of the analysis of cell-free mutated tumor DNA as novel diagnostic [27], predictive [31-33] and prognostic [31, 32, 34-37] biomarker for pancreatic cancer.

What takes our study apart is the use of discriminatory multi-target KRAS ddPCR assays [30] to directly identify KRAS SNVs without performing previous tumor NGS. These assays have higher sensitivity compared to many available NGS-based assays [29]. Moreover, the input volume of $4 \mathrm{ml}$ plasma for cfDNA extraction might have contributed to the comparably higher sensitivity of our assays compared to previous reports [26]. In comparison to more sophisticated NGS panels specifically developed for cfDNA analysis [29, 38], multi-target ddPCR assays are associated with much lower assay costs, allowing for the serial analysis through clinical course analogous to CA 19-9 levels. Using these assays, our $\mathrm{cfKR} A S^{\mathrm{mut}}$ detection rate in the cohort was similar to other published data for patients following PDAC resection [20,31,36]. A very high concordance rate between tumor tissue und detected cfDNA KRAS SNVs further validates our approach. 
In our cohort, detection of $\mathrm{cf} K R A S^{\mathrm{mut}}$ in the first postoperative sample alone did not significantly correlate with survival (data not shown), while elevated CA 19-9 levels at first presentation were associated with poor

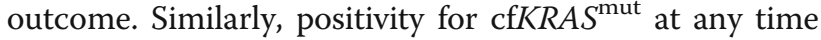
point above assay threshold alone was not significantly associated with survival. However, when choosing a more stringent $\operatorname{cf} K R A S^{\text {mut }}$ cut-off or when analyzing dynamic changes (increase vs non-increase), $\operatorname{cfKRAS^{\text {mut}}}$ was strongly associated with survival and outperformed CA 19-9 levels for association with relapse and OS, highlighting the importance of identifying clinically validated cut-offs for cfDNA analysis [39-41] and also underlining the limitations associated with analyzing a small patient cohort.

One main finding of our analysis was that $\mathrm{cfKR} A S^{\mathrm{mut}}$ positivity and CA 19-9 elevation are only partially overlapping and that combining both parameters identifies a larger cohort of patient with poor outcome. Several studies have suggested integration of established and experimental protein biomarkers with cfDNA analysis for pancreatic cancer early diagnostics [27, 28, 42-44], identification of minimal residual disease [45] and molecular monitoring for advanced disease [26, 41]. Our approach is focused on clinical applicability and feasibility through integration of the two relatively easy-to-assess biomarkers cfKRAS ${ }^{\text {mut }}$ analysis and CA 19-9. Notably, a recent large multi-center case-control study did not find a benefit if $\mathrm{cfKRAS} S^{\text {mut }}$ analysis as compared to CA 19-9 analysis in pancreatic cancer patients across all stages of disease [26]. In this study, however, reported detection rates of $\mathrm{cfKRAS} S^{\mathrm{mut}}$ were overall lower than in our study, probably due to differences in assay technology. Our data suggest that CA 19-9 and cfKRAS ${ }^{\text {mut }}$ levels each have their own distinct advantages and disadvantages and that integrating them for analysis might be superior to analyzing them individually, the question of how best to integrate both biomarkers for clinical practice remains challenging, which is also illustrated by the analysis of single patient's disease course in our cohort.

A major limitation of our study is the small cohort size, which makes it difficult to define clinically relevant cut-off levels forcfKRAS ${ }^{\text {mut }}$ and to optimize integration of CA 19-9 with cfKRAS ${ }^{\text {mut }}$. However, despite these limitations, our study also points to the potential of clinically further developing $\mathrm{cfKRAS} S^{\mathrm{mut}}$ as prognostic and predictive biomarkers for the management of resectable PDAC. Most importantly, further studies will have to explore the potential of biomarker-based therapeutic intervention for pancreatic cancer. Systemic treatment options for PDAC are limited to a small number of combination chemotherapy regimens $[46,47]$ and some recent developments in personalized treatment based on molecular profiling [14]. A switch of adjuvant chemotherapy regimen based on molecular monitoring appears feasible yet will need extensive clinical validation in interventional trials, especially since established adjuvant treatment standards took so many years to establish.

In summary our study proposed a clinically feasible approach to assay cfKRAS ${ }^{\text {mut }}$ together with CA 19-9 in patients following curative resection of PDAC. Through combination of both markers, patients could be better stratified in terms of relapse risk and overall prognosis.

\section{Conclusion}

The integrated longitudinal analysis of $\mathrm{cfKRAS^{mut }}$ and CA 19-9 levels holds potential for the molecular monitoring of patients following resection of PDAC. Larger cohorts and prospective trials are required to establish clinically relevant cut-off levels and to better unravel the relationships between biomarker dynamics and clinical relapse.

\section{Supplementary Information}

The online version contains supplementary material available at https://doi. org/10.1186/s12885-020-07736-x.

\begin{abstract}
Additional file 1: Supplemental Figure 1. Univariate survival analyses for established clinicopathologic variables. A, B) Kaplan-Meier estimates of RFS (A) and OS (B) for PDAC patients stratified in two groups: patients with R1 vs. R0 resection. (C, D) Kaplan-Meier estimates of RFS (C) and OS (D) for PDAC patients stratified by postoperative CA 19-9 levels: elevated (> $36 \mathrm{U} / \mathrm{mL}$ ) versus normal $(\leq 36 \mathrm{U} / \mathrm{mL}$ ). (E, F) Kaplan-Meier estimates of RFS (E) and OS (F) for resected PDAC patients with/without adjuvant chemotherapy. OS, overall survival; RFS, recurrence-free survival; PDAC, pancreatic ductal adenocarcinoma. Univariate analyses were performed using the log-rank test. Supplemental Figure 2. Association of cfKRASmut with survival endpoints and clinicopathologic variables. (A, B) KaplanMeier estimates of RFS (A) and OS (B) for patients following curative resection of PDAC with versus without cfKRAS mut cut-off level of $>0.5 \%$ variant allele frequency (VAF) at any time point during study period. (C, $D, E) c^{\prime} K_{R A S}{ }^{m u t}(C)$, total cell-free DNA (D) and CA 19-9 levels (E) in untreated resected PDAC patients were compared to resection margin. OS, overall survival; RFS, recurrence-free survival; PDAC, pancreatic ductal adenocarcinoma
\end{abstract}

Additional file 2: Table S1. Primer and probe sequences for KRAS $\mathrm{ddPCR}$ assays. Table S2. Overall survival analysis by clinico-pathologic variables. Table S3. Recurrence-free survival analysis by clinicopathologic variables. Table S4. Comparison between tissue analyses and CfDNA ddPCR

\section{Abbreviations}

cfDNA: Cell-free DNA; ffKRAS $^{\text {mut }}$ : Mutated cell-free plasma KRAS; ctDNA: Tumor-derived cell-free nucleic acids; ddPCR: Droplet digital PCR; IDT: Integrated DNA Technologies; IRB : Institutional review board; HR: Hazard ratio; LOB: Limit of blank; LOD: Limit of detection; mFOLFIRINOX: 5-fluorouracil, irinotecan, oxaliplatin, leucovorin; NGS: Next generation sequencing; OS: Overall survival; PCR: Polymerase chain reaction; PDAC: Pancreatic ductal adenocarcinoma; RFS: Recurrence-free survival; SNV: Single nucleotide variants

\section{Acknowledgements}

S.H. was recipient of a DGHO-GMIHO thesis fellowship. Ralph Fritsch is guarantor of this work and, as such, had full access to all data in the study and takes responsibility for the integrity of the data and the accuracy of the data analysis. 


\section{Authors' contributions}

$\mathrm{SH}$ collected, analyzed and interpreted the patient data. SH, DA and RF were major contributors in writing the manuscript. SH, MB, UW and RF conceptualized the study. RF supervised the data collection, analysis and writing of the manuscript. JH, DA, MF, RK, UP, FS, NB and JD contributed to data collection and manuscript edition. All authors read and approved the final manuscript.

\section{Funding}

This work was supported by Foerdergesellschaft Stiftung Tumorbiologie, Freiburg, Germany, and a Comprehensive Cancer Center Freiburg (CCCF) Research Grant, by the Deutsche Forschungsgemeinschaft (DFG CRC-850 subprojects C9 to R.F. and M.B. and Z1 to M. B. and by the German Federal Ministry of Education and Research (BMBF) within the framework of the e:Med research and funding concept CoNfirm (FKZ 01ZX1708F to M. B.). Funders had no role in the design, execution, analysis or publication of the study.

\section{Availability of data and materials}

The datasets used and/or analyzed during the current study are available from the corresponding author on reasonable request.

\section{Ethics approval and consent to participate}

Local institutional review board (IRB) approved all study procedures (EK48/ 18). All patients provided written informed consent for sample collection and analysis.

\section{Consent for publication}

Not applicable.

\section{Competing interests}

The authors declare that they have no competing interests.

\section{Author details}

'Department of Medicine I (Hematology, Oncology and Stem Cell Transplantation), Freiburg University Medical Center, Freiburg, Germany. ${ }^{2}$ Department of Medical Oncology and Hematology, Zurich University Hospital, Raemistrasse 100, 8091 Zürich, Switzerland. ${ }^{3}$ Department of Surgery, Freiburg University Medical Center, Freiburg, Germany. ${ }^{4}$ German Cancer Consortium (DKTK), partner site Freiburg, German Cancer Research Center (DKFZ), Heidelberg, Germany. ${ }^{5}$ Comprehensive Cancer Center Freiburg (CCCF), Medical Center - University of Freiburg, Faculty of Medicine, University of Freiburg, Freiburg, Germany. ${ }^{6}$ Institute of Medical Bioinformatics and Systems Medicine, University Medical Center Freiburg, Faculty of Medicine, University of Freiburg, Freiburg, Germany. ${ }^{7}$ German Cancer Research Center (DKFZ), Heidelberg, Germany.

Received: 16 August 2020 Accepted: 15 December 2020 Published online: 11 January 2021

\section{References}

1. Rawla P, Sunkara T, Gaduputi V. Epidemiology of pancreatic Cancer: global trends, etiology and risk factors. World J Oncol. 2019;10(1):10-27.

2. Bridgewater J, Lopes A, Wasan H, Malka D, Jensen L, Okusaka T, Knox J, Wagner D, Cunningham D, Shannon J, et al. Prognostic factors for progression-free and overall survival in advanced biliary tract cancer. Ann Oncol. 2016;27(1):134-40.

3. Huang $L$, Jansen $L$, Balavarca $Y$, Babaei $M$, van der Geest $L$, Lemmens $V$, Van Eycken L, De Schutter H, Johannesen TB, Primic-Žakelj M, et al. Stratified survival of resected and overall pancreatic cancer patients in Europe and the USA in the early twenty-first century: a large, international populationbased study. BMC Med. 2018;16(1):125.

4. Wittel UA, Lubgan D, Ghadimi M, Belyaev O, Uhl W, Bechstein WO, Grützmann R, Hohenberger WM, Schmid A, Jacobasch L, et al. Consensus in determining the resectability of locally progressed pancreatic ductal adenocarcinoma - results of the Conko-007 multicenter trial. BMC Cancer. 2019;19(1):979.

5. Chakraborty S, Singh S. Surgical resection improves survival in pancreatic cancer patients without vascular invasion- a population based study. Ann Gastroenterol. 2013;26(4):346-52.
6. Katz MH, Wang H, Fleming JB, Sun CC, Hwang RF, Wolff RA, Varadhachary G, Abbruzzese JL, Crane CH, Krishnan S, et al. Long-term survival after multidisciplinary management of resected pancreatic adenocarcinoma. Ann Surg Oncol. 2009;16(4):836-47.

7. Oettle H, Post S, Neuhaus P, Gellert K, Langrehr J, Ridwelski K, Schramm H, Fahlke J, Zuelke C, Burkart C, et al. Adjuvant chemotherapy with gemcitabine vs observation in patients undergoing curative-intent resection of pancreatic cancer: a randomized controlled trial. Jama. 2007;297(3):267-77.

8. Smeenk HG, van Eijck CH, Hop WC, Erdmann J, Tran KC, Debois M, van Cutsem E, van Dekken $\mathrm{H}$, Klinkenbijl JH, Jeekel J. Long-term survival and metastatic pattern of pancreatic and periampullary cancer after adjuvant chemoradiation or observation: long-term results of EORTC trial 40891. Ann Surg. 2007;246(5):734-40.

9. Sperti C, Pasquali C, Piccoli A, Pedrazzoli S. Recurrence after resection for ductal adenocarcinoma of the pancreas. World J Surg. 1997;21(2): 195-200.

10. Raut CP, Tseng JF, Sun CC, Wang H, Wolff RA, Crane CH, Hwang R, Vauthey J-N, Abdalla EK, Lee JE, et al. Impact of resection status on pattern of failure and survival after pancreaticoduodenectomy for pancreatic adenocarcinoma. Ann Surg. 2007;246(1):52-60.

11. Conroy T, Hammel P, Hebbar M, Ben Abdelghani M, Wei AC, Raoul J-L, Choné L, Francois E, Artru P, Biagi JJ, et al. FOLFIRINOX or Gemcitabine as Adjuvant Therapy for Pancreatic Cancer. N Engl J Med. 2018;379(25):2395406.

12. Ying H, Dey P, Yao W, Kimmelman AC, Draetta GF, Maitra A, DePinho RA. Genetics and biology of pancreatic ductal adenocarcinoma. Genes Dev. 2016;30(4):355-85.

13. Yao W, Maitra A, Ying $H$. Recent insights into the biology of pancreatic cancer. eBioMedicine. 2020;53:102655.

14. Golan T, Hammel P, Reni M, Van Cutsem E, Macarulla T, Hall MJ, Park JO, Hochhauser D, Arnold D, Oh DY, et al. Maintenance Olaparib for Germline BRCA-mutated metastatic pancreatic Cancer. N Engl J Med. 2019;381(4):31727.

15. Grinshpun A, Zarbiv Y, Roszik J, Subbiah V, Hubert A. Beyond KRAS: practical molecular targets in pancreatic adenocarcinoma. Case Rep Oncol. 2019; 12(1):7-13.

16. Aguirre AJ. Oncogenic NRG1 Fusions: A New Hope for Targeted Therapy in Pancreatic Cancer. Clin Cancer Res. 2019;25(15):4589-91.

17. Khater F, Langlois S, Cassart P, Roy AM, Lajoie M, Healy J, Richer C, St-Onge P, Piché N, Perreault S, et al. Recurrent somatic BRAF insertion (p.V504_ R506dup): a tumor marker and a potential therapeutic target in pilocytic astrocytoma. Oncogene. 2019;38(16):2994-3002.

18. Amin S, Baine M, Meza J, Lin C. The impact of neoadjuvant and adjuvant immunotherapy on the survival of pancreatic cancer patients: a retrospective analysis. BMC Cancer. 2020;20(1):538.

19. Alix-Panabières C, Pantel K. Clinical applications of circulating tumor cells and circulating tumor DNA as liquid biopsy. Cancer Discov. 2016; 6(5):479-91.

20. Bettegowda C, Sausen M, Leary RJ, Kinde I, Wang Y, Agrawal N, Bartlett BR, Wang H, Luber B, Alani RM, et al. Detection of circulating tumor DNA in early- and late-stage human malignancies. Sci Transl Med. 2014;6(224) 224ra224.

21. Diaz LA Jr, Bardelli A. Liquid biopsies: genotyping circulating tumor DNA. J Clin Oncol. 2014:32(6):579-86.

22. Macías M, Alegre E, Díaz-Lagares A, Patiño A, Pérez-Gracia JL, Sanmamed M, López-López R, Varo N, González A. Liquid biopsy: from basic research to clinical practice. Adv Clin Chem. 2018;83:73-119.

23. Gormally E, Caboux E, Vineis P, Hainaut P. Circulating free DNA in plasma or serum as biomarker of carcinogenesis: practical aspects and biological significance. Mutat Res. 2007;635(2-3):105-17.

24. Kato $S$, Janku F. Cell-free DNA as a novel marker in cancer therapy. Biomark Med. 2015:9(7):703-12.

25. Meng P, Wei J, Geng Y, Chen S, Terpstra MM, Huang Q, Zhang Q, Su Z, Yu $W$, Su M, et al. Targeted sequencing of circulating cell-free DNA in stage II-II resectable oesophageal squamous cell carcinoma patients. BMC Cancer. 2019;19(1):818.

26. Le Calvez-Kelm F, Foll M, Wozniak MB, Delhomme TM, Durand G, Chopard P, Pertesi M, Fabianova E, Adamcakova Z, Holcatova I, et al. KRAS mutations in blood circulating cell-free DNA: a pancreatic cancer case-control. Oncotarget. 2016;7(48):78827-40. 
27. Cohen JD, Javed AA, Thoburn C, Wong F, Tie J, Gibbs P, Schmidt CM, Yip-Schneider MT, Allen PJ, Schattner M, et al. Combined circulating tumor DNA and protein biomarker-based liquid biopsy for the earlier detection of pancreatic cancers. Proc Natl Acad Sci USA. 2017;114(38): 10202-7.

28. Cohen JD, Li L, Wang Y, Thoburn C, Afsari B, Danilova L, Douville C, Javed AA, Wong F, Mattox A, et al. Detection and localization of surgically resectable cancers with a multi-analyte blood test. Science. 2018;359(6378):926-30.

29. Zill OA, Greene C, Sebisanovic D, Siew LM, Leng J, Vu M, Hendifar AE, Wang Z, Atreya CE, Kelley RK, et al. Cell-free DNA next-generation sequencing in Pancreatobiliary carcinomas. Cancer Discov. 2015;5(10):1040-8.

30. Hussung S, Follo M, Klar RFU, Michalczyk S, Fritsch K, Nollmann F, Hipp J, Duyster J, Scherer F, von Bubnoff N, et al. Development and clinical validation of discriminatory multitarget digital droplet PCR assays for the detection of hot spot KRAS and NRAS mutations in cell-free DNA. J Mol Diagnostics. 2020;22(7):943-56.

31. Bernard V, Kim DU, San Lucas FA, Castillo J, Allenson K, Mulu FC, Stephens BM, Huang J, Semaan A, Guerrero PA, et al. Circulating Nucleic Acids Are Associated With Outcomes of Patients With Pancreatic Cancer. Gastroenterology. 2019;156(1):108-18 e104.

32. Watanabe F, Suzuki K, Tamaki S, Abe I, Endo Y, Takayama Y, Ishikawa H, Kakizawa $\mathrm{N}$, Saito M, Futsuhara K, et al. Longitudinal monitoring of KRAS-mutated circulating tumor DNA enables the prediction of prognosis and therapeutic responses in patients with pancreatic cancer. PLoS One. 2019;14(12):e0227366.

33. Sausen M, Phallen J, Adleff V, Jones S, Leary RJ, Barrett MT, Anagnostou V, Parpart-Li S, Murphy D, Kay Li Q, et al. Clinical implications of genomic alterations in the tumour and circulation of pancreatic cancer patients. Nat Commun. 2015:6:7686

34. Cheng H, Liu C, Jiang J, Luo G, Lu Y, Jin K, Guo M, Zhang Z, Xu J, Liu L, et al. Analysis of ctDNA to predict prognosis and monitor treatment responses in metastatic pancreatic cancer patients. Int J Cancer. 2017; 140(10):2344-50.

35. Patel H, Okamura R, Fanta P, Patel C, Lanman RB, Raymond VM, Kato S, Kurzrock R. Clinical correlates of blood-derived circulating tumor DNA in pancreatic cancer. J Hematol Oncol. 2019;12(1):130.

36. Pietrasz D, Pécuchet N, Garlan F, Didelot A, Dubreuil O, Doat S, Imbert-Bismut F, Karoui M, Vaillant JC, Taly V, et al. Plasma circulating tumor DNA in pancreatic Cancer patients is a prognostic marker. Clin Cancer Res. 2017;23(1):116-23.

37. Gall TMH, Belete $S$, Khanderia E, Frampton AE, Jiao LR. Circulating tumor cells and cell-free DNA in pancreatic ductal adenocarcinoma. Am J Pathol. 2019;189(1):71-81.

38. Malapelle U, Mayo de-Las-Casas C, Rocco D, Garzon M, Pisapia P, JordanaAriza N, Russo M, Sgariglia R, De Luca C, Pepe F, et al. Development of a gene panel for next-generation sequencing of clinically relevant mutations in cell-free DNA from cancer patients. Br J Cancer. 2017;116(6):802-10.

39. Crowley E, Di Nicolantonio F, Loupakis F, Bardelli A. Liquid biopsy: monitoring cancer-genetics in the blood. Nat Rev Clin Oncol. 2013;10(8):472-84.

40. Fleischhacker M, Schmidt B. Circulating nucleic acids (CNAs) and cancer--a survey. Biochim Biophys Acta. 2007;1775(1):181-232.

41. Bronkhorst AJ, Ungerer $V$, Holdenrieder $\mathrm{S}$. The emerging role of cell-free DNA as a molecular marker for cancer management. Biomol Detect Quantif. 2019;17:100087.

42. Nazli O, Bozdag AD, Tansug T, Kir R, Kaymak E. The diagnostic importance of CEA and CA 19-9 for the early diagnosis of pancreatic carcinoma. Hepato-gastroenterology. 2000;47(36):1750-2.

43. Capello M, Bantis LE, Scelo G, Zhao Y, Li P, Dhillon DS, Patel NJ, Kundnani $\mathrm{DL}$, Wang $\mathrm{H}$, Abbruzzese $J$, et al. Sequential Validation of Blood-Based Protein Biomarker Candidates for Early-Stage Pancreatic Cancer. J Natl Cancer Institute. 2016;109(4).

44. Herreros-Villanueva M, Bujanda L. Non-invasive biomarkers in pancreatic cancer diagnosis: what we need versus what we have. Ann Transl Med. 2016;4(7):134

45. Chae YK, Oh MS. Detection of minimal residual disease using ctDNA in lung Cancer: current evidence and future directions. J Thoracic Oncol. 2019;14(1):16-24.
46. Conroy T, Desseigne F, Ychou M, Bouché O, Guimbaud R, Bécouarn Y Adenis A, Raoul J-L, Gourgou-Bourgade S, de la Fouchardière C, et al. FOLFIRINOX versus Gemcitabine for Metastatic Pancreatic Cancer. N Engl J Med. 2011;364(19):1817-25.

47. Von Hoff DD, Ervin T, Arena FP, Chiorean EG, Infante J, Moore M, Seay T, Tjulandin SA, Ma WW, Saleh MN, et al. Increased Survival in Pancreatic Cancer with nab-Paclitaxel plus Gemcitabine. N Engl J Med. 2013;369(18): 1691-703.

\section{Publisher's Note}

Springer Nature remains neutral with regard to jurisdictional claims in published maps and institutional affiliations.

\section{Ready to submit your research? Choose BMC and benefit from:}

- fast, convenient online submission

- thorough peer review by experienced researchers in your field

- rapid publication on acceptance

- support for research data, including large and complex data types

- gold Open Access which fosters wider collaboration and increased citations

- maximum visibility for your research: over $100 \mathrm{M}$ website views per year

At BMC, research is always in progress.

Learn more biomedcentral.com/submissions 